\title{
Metformin Ameliorates Oxidative Stress Induced by Diabetes Mellitus and Hepatocellular Carcinoma in Rats
}

\author{
Maysa Ahmed Mobasher*1,2, Hala Galal El-Tantawi ${ }^{3}$, Karim Samy El-Said ${ }^{4}$
}

\begin{abstract}
Background: Several studies have found an association between Diabetes mellitus (DM) and an increased risk for hepatocellular carcinoma (HCC). Evidence suggests that Metformin (Met) may have a therapeutic and protective effect against both DM and HCC. Therefore, the aim of this study was to evaluate the antioxidant effect of Met against DM and HCC-induced oxidative stress in rat model.

Methods: Forty-two male albino rats were randomly divided into six groups. Group $1(\mathrm{Gp} 1)$ was the control group, Gp2 received an intraperitoneal (i.p.) injection with streptozotocin (STZ), Gp3 was injected i.p. with diethyl nitrosamine (DEN), Gp4 received an oral administration of Met, Gp5 and Gp6 received the same injections as $\mathrm{Gp} 2$ and $\mathrm{Gp} 3$, respectively, then received an additional injection of Met. Oxidative stress biomarkers, including superoxide dismutase (SOD), catalase (CAT), reduced glutathione (GSH) and malondialdehyde (MDA), were examined. Furthermore, biochemical parameters including liver function tests were assessed. Histopathological and immunohistochemical alterations of the liver were also examined. Results: Our results demonstrate that Gp2 and Gp3 had significant signs of liver dysfunction and had elevated levels of MDA and reduced levels of SOD, CAT, and GSH. Additionally, Gp2 and Gp3 showed significant alterations in the liver architecture shown by high PCNA and caspase-3 expression. In the Gp5 and Gp6, treatment with Met showed an improvement in liver function, oxidative stress biomarkers, and reduced histopathological changes in hepatocytes.

Conclusions: This study offers insight into the potential for Metformin as a novel therapeutic against the oxidative stress induced by DM or HCC.
\end{abstract}

Keywords: Diabetes Mellitus, Diethyl nitrosamine, Hepatocellular Carcinoma, Metformin, Streptozotocin.

\section{Introduction}

Diabetes mellitus (DM) is one of the most widely recognized chronic diseases in almost all nations and classified as the $9^{\text {th }}$ major cause of death worldwide (1). DM has been strongly linked with the non-alcoholic fatty-liver disease (NAFLD) and many other metabolic disturbances (2). It is worthy to mention that DM is an important risk factor for the development of many types of malignancies (3). Cancer alone represents the most significant cause of death worldwide and eliminates around 6 million lives each year (4). In particular, hepatocellular carcinoma (HCC), the malignant liver cancer represents the $5^{\text {th }}$ common cancer and the $3^{\text {rd }}$ cause of cancer-related mortality worldwide (5) in accordance with the World Health Organization (WHO). Previous research has shown that obesity and its related metabolic abnormalities, particularly DM, significantly, increase the danger of HCC development (6) due to the production of hyperinsulin-like growth factor 1 (IGF-1) and oxidative stress (7). Diabetic patients develop several complications including non-alcoholic fatty liver that contribute in progressive liver diseases such 
as cirrhosis and cancer (7). Despite substantial advancement in understanding the clinical relation between DM and HCC, further injurious mechanisms responsible for this cellular damage remain unknown. Indeed, this lack of knowledge, attracts many researchers to elucidate this relationship (8).

Simultaneously, the type and prescribed dose of the utilized antidiabetic drugs are also implicated in influencing the danger of HCC development (9). Thus, emerging from these tremendous contributions between DM and HCC, reliable management of DM is suggested (8).

Insulin resistance in type $2 \mathrm{DM}(\mathrm{T} 2 \mathrm{DM})$ is a key pathological feature for HCC oncogenesis. It has been reported that insulin resistance may have a potential role in hepatocarcinogenesis in patients with T2DM or prediabetes infected with hepatitis B virus (HBV) (6). Reportedly, hyperinsulinemia upregulates the levels of insulin-like growth factors (IGFs), and abnormal activation of the IGF/IGF-1 receptor (IGF-1R) axis plays an important role in the development of different types of human malignancies, involving HCC (10). Therefore, these data advocated that targeting insulin resistance could be an applicable approach for repressing the incidence of obesityrelated $\mathrm{HCC}$.

Furthermore, the potential role of oxidative stress in the production of tissue damage in DM had been discussed. In addition, oxidative stress is the primary driving force of HCC development which predisposes to hepatocarcinogenesis. Under normal physiological state, a balance between reactive oxygen species (ROS) generation and oxidative defenses mechanism exists in all cells, which modulated by endogenous antioxidant enzymes as superoxide dismutase (SOD), catalase (CAT), and glutathione peroxidase (Gpx1). During the course of DM and HCC, the level of oxidant biomarkers such as MDA and G-S-S-G increased due to the release of elevated levels of both reactive oxygen species and nitrogen species (ROS and RNS, respectively) (11) together with reductions in the expression of antioxidants enzymes levels. Therefore, treatment with antioxidant agents could decrease the oxidative stress state, which in turn ameliorates the severity of such diseases (12).
Metformin (Met) is a first-line DM therapy, which decreases the glucose production in the liver by increasing the body's sensitivity to insulin (13). Previous pharmacoepidemiologic study revealed that Met treatment seems to prevent hepatocellular transformation, diminishing the danger of HCC development to degrees parallel to that of non-diabetic patients (14). The previous findings of De Peralta et al, (15) reported that the prevalence of HCC, as well as fibrotic and inflammatory markers levels were reduced when Met treatment begun at the first signs of fibrosis. Notwithstanding, HCC burden was unchanged when Met treatment begun at the first signs of cirrhosis (15). Notably, it has been reported that Met might operate to inhibit tumorigenesis through both the insulin-dependent and insulinindependent basic mechanisms (16). Additionally, it has been shown that Met has potent antioxidant properties besides its therapeutic effect against DM (17). Based on that, this study aimed to investigate and underlie the antioxidant potency of Met versus the oxidative stress induced by STZ and DEN in $\mathrm{DM}$ and HCC rats, respectively and to clarify the connection between diabetes, oxidative stress, and cancer morbidity or cancer prognosis. Results emerging from this work will help to control the disease development and provide an efficient therapy.

\section{Materials and methods}

\section{Chemicals}

Streptozotocin (STZ) and diethylnitrosamine (DEN) were purchased from Sigma (St. Louis, MO, USA). DEN was dissolved in saline. All biochemical kits were obtained from the Biodiagnostic company (Cairo-Egypt). For immunohistochemical preparation, the primary and secondary antibodies were purchased from Dako Company (Glostrup, Denmark).

\section{Rats}

Forty-two male Sprague Dawley albino rats, (100 $\pm 5 \mathrm{~g}$ ) were obtained from the National Research Center (NRC, Cairo, Egypt), housed randomly as 6 rats per cage, in $12 \mathrm{~h} / 12 \mathrm{~h}$ dark/light cycle under standard conditions of temperature and humidity. Animals were carefully observed every day and 
their body weights were recorded, while food consumption and water intakes were measured precisely every week to evaluate any signs of toxicity or abnormality during the experiment. Animal experimentations were approved by the ethics committee at the Faculty of Science, Tanta University (Egypt), and were conducted according to their accepted ethical guidelines for the Animal Ethics Committee (No. IACUC-SCITU-0084).

\section{DM and HCC inductions}

For DM induction, rats received a single intraperitoneal (i.p) dose of STZ $(80 \mathrm{mg} / \mathrm{kg})$ (18). For HCC induction, rats were i.p injected with DEN (50 mg/kg) once weekly for 7 weeks (15). For Met treatment, rats were treated by gavage with $150 \mathrm{mg} / \mathrm{kg}$ every other day for 100 days (19) (Fig. 1).

\section{Experimental design}

All rats were clinically healthy. The animals were divided randomly into six equal groups $(n=7)$ as the following: Gp1 was served as normal untreated control rats, $\mathrm{Gp} 2$ was injected with STZ, Gp3 was injected with DEN, Gp4 was orally administrated with Met, Gp5 was injected with STZ and treated with Met and Gp6 was injected with DEN and treated with Met. Met was orally administered after blood glucose level reached up to $250 \mathrm{mg} / \mathrm{dl}$ in the DM model and at 50 days' post-DEN-injection in the HCC model (Fig. 1).

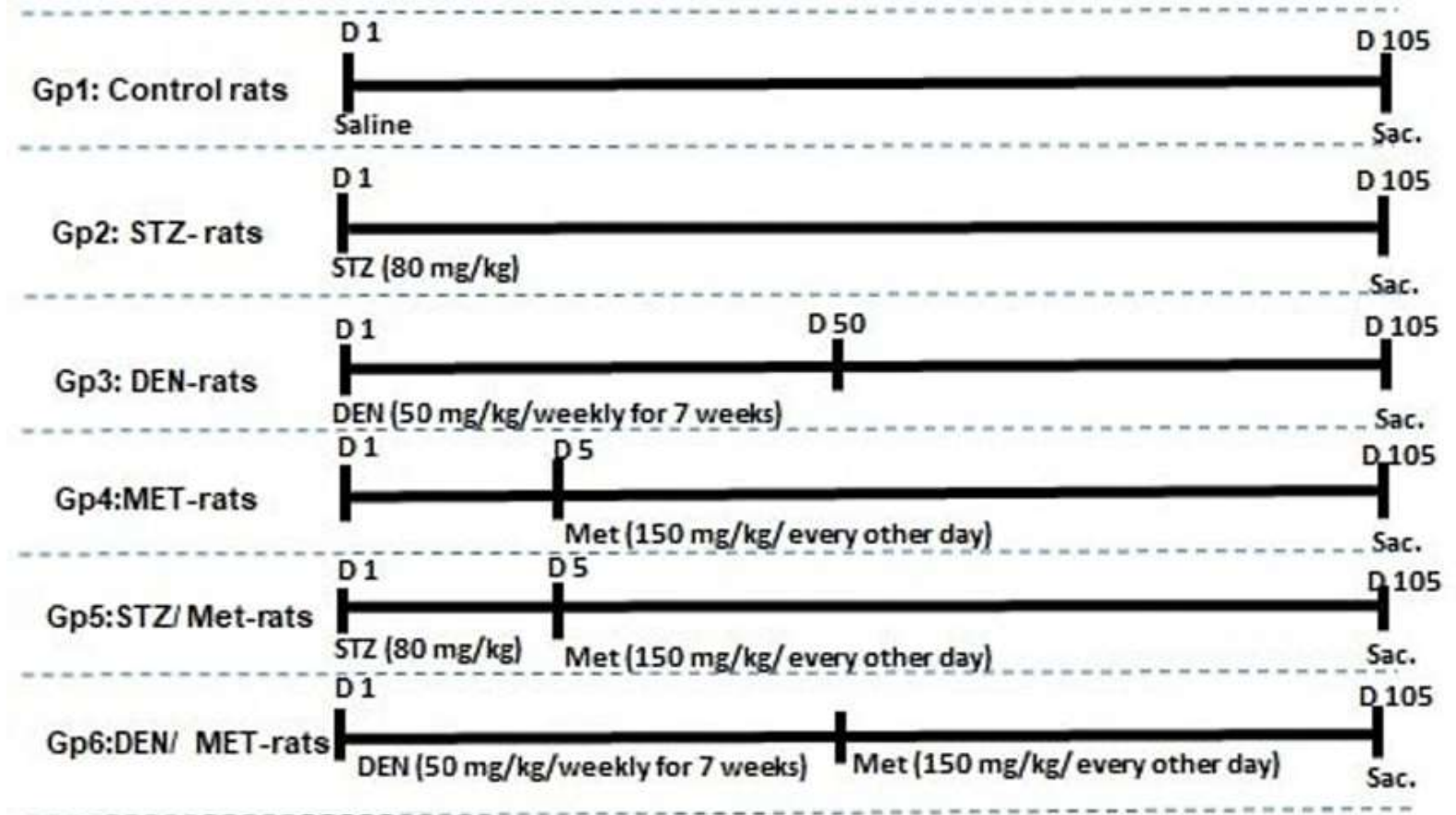

Fig. 1. Experimental design. STZ: Streptozotocin $(80 \mathrm{mg} / \mathrm{kg}$ once); Met: Metformin ( $150 \mathrm{mg} / \mathrm{Kg} /$ each other day); DEN: Diethylnitrosamine (50 mg/Kg/ once per week for 7 weeks). Gp1 were normal untreated control rats, Gp2 was injected with STZ, Gp3 was injected with DEN, Gp4 was administered Met via gavage, Gp5 was injected with STZ and treated with Met, and Gp6 was injected with DEN and treated with Met.

\section{Samples}

At the end of the experiment, by the day 105 , all rats were sacrificed under ethyl ether anesthesia and cadavers were burned in animal incinerators under the supervision of the Faculty of Science, Tanta University. Gross examinations were performed macroscopically on all groups during sacrifice. The percentages of absolute and relative organ weights (organ $w t / b . w t \times 100)$ of all rats were taken after organs being necropsied.

Blood samples were collected from arterial blood vessels and heart chambers for hematological examinations, and sera were buffer saline (PBS). The resulting supernatants were used for biochemical analysis. 
Furthermore, samples from liver tissues were collected in buffered formalin for histological and immunohistochemical investigations.

\section{Hematological and biochemical profiling}

Hemoglobin ( $\mathrm{Hb}$ ) levels, hematocrit (Hct\%), platelets count, the total count of red blood cells (RBCs), white blood cells (WBCs) and differential count were detected by using auto hematology analyzer (BC-3200, Mindray, China). Alanine aminotransferase (ALT) and aspartate aminotransferase (AST) were determined as described (20). Alkaline phosphatase (ALP), arginase activity, and total protein (TP) were assessed as previously described (21-23), respectively. Albumin was assayed as the method described by Burtis and Bruns (24). Total bilirubin (TB) was determined according to Walter and Gerade (25). Serum cholesterol, triglycerides, and HDL-cholesterol were determined using quantitative kit based on the previously described methods (26-28), respectively. Low-density lipoprotein cholesterol (LDL) was calculated according to Friedewald et al, (29) as follows: $\mathrm{LDL}=$ total cholesterolHDL- VLDL. Superoxide dismutase (SOD) and catalase activities were determined as described (30, 31), respectively. Levels of reduced glutathione (GSH) were measured according to Paglia and Valentine (32). Malondialdehyde (MDA) was determined based on methods of $\mathrm{Li}$ and Chow, briefly, samples were deproteinized and then mixed with Thiobarbituric acid (TBA) $0.67 \%(\mathrm{~W} / \mathrm{V})$, and the absorbance was read at $532 \mathrm{~nm}$ against a blank (33).

\section{Histological staining}

Tissue specimens of the liver were harvested and fixed in $10 \%$ formalin. Paraffin blocks were prepared after completing the tissue processing in different grades of alcohol and xylene. Sections $(5 \mu \mathrm{m})$ were prepared from paraffin blocks using microtome, stained with hematoxylin and eosin, and observed under a light microscope (Optica light microscope (B350)) to examine gross cellular damage (34).

\section{Immunohistochemical staining for PCNA and caspase-3 detection}

The liver tissues of Sprague Dawley albino rats were fixed in $10 \%$ buffered formalin for 18 $24 \mathrm{hr}$ and transferred to $70 \%$ ethanol overnight. All temperatures for processing and embedding were kept under $60{ }^{\circ} \mathrm{C}$. The super sensitive biotin-streptavidin method for immunohistochemical localization was performed. Negative control slides from each of the same tissues were incubated with a control antibody. The blocking, primary, and control antibodies were diluted with a diluent composed of $1 \%$ bovine serum albumin in I X automation buffer.

\section{Statistical analysis}

Data was presented as mean \pm SD and were analyzed using one-way analysis of variance (ANOVA) followed by Tukey's test and $p<0.05$ were statistically significant.

\section{Results}

Treatment with Met in rats with DM decreased body weight and glucose levels

The experimental design and the time course of treatments in all groups under study are shown in Figures $1 \& 2$ shows that total body weight until week 7 was similar in all groups of rats. Likewise, rats receiving STZ (Gp2), Met (Gp4) and the DEN/Met group (Gp6) showed similar changes in their body weight along weeks 8 and 14 of the treatment. However, at this timeperiod rats injected with STZ and treated with Met (Gp5) showed a significant decrease in the total body weight when compared with the control (Gp1).

After 3 weeks of STZ injection, glucose levels were increased in the diabetic group (Gp2) up to $250 \mathrm{mg} / \mathrm{dl}$; however, treatment with Met post-DM induction decreased glucose levels dramatically, which returned close to the normal level by week 8 . Group of rats injected with DEN alone (Gp3) did not show any significant changes in the glucose levels during the full time of the experiment. 


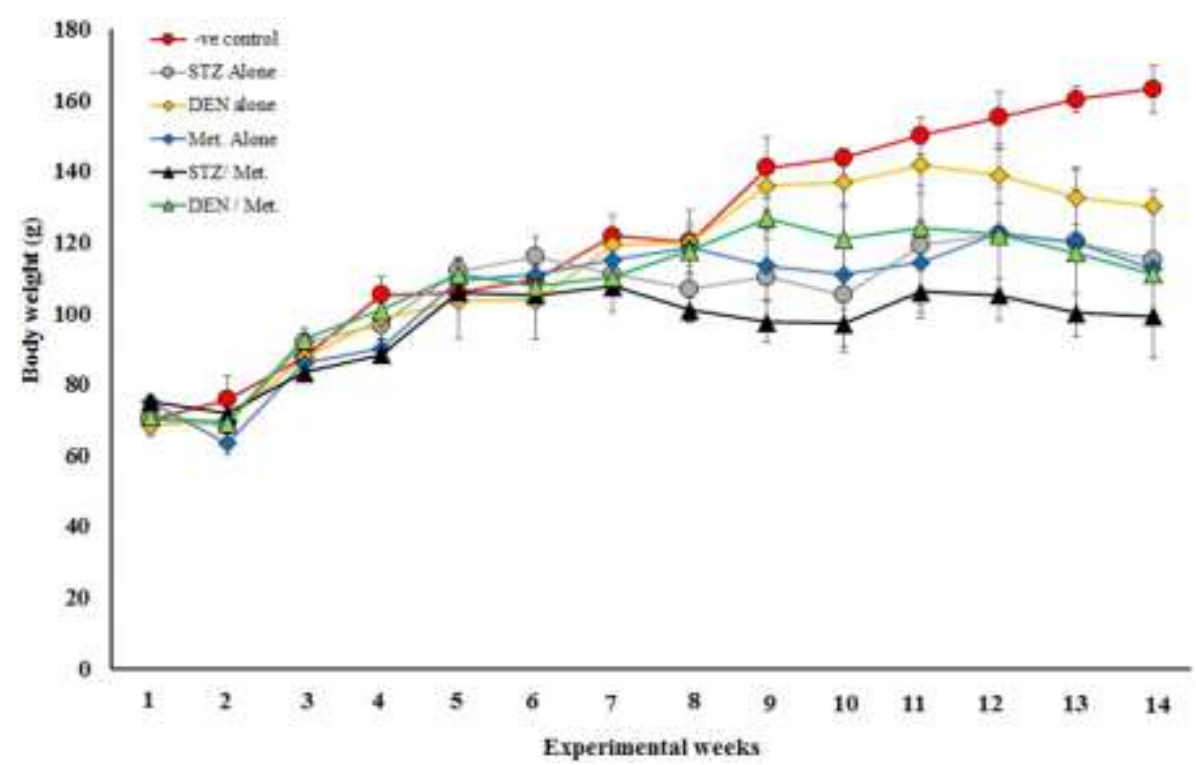

Fig. 2. Changes in body weight in the different groups under the study. Treatment with STZ, DEN, Met, or their combinations post 14 weeks led to differences in the body weights of rats.

\section{Met did not significantly alter the hematological parameters.}

Hematological analysis of all groups under study showed that only the group of rats injected with DEN (Gp3) revealed an increase in the total white blood cells (WBCs) when compared to the other groups. Concomitant to this increase in WBCs, the percentage $(\%)$ of neutrophils and monocytes were increased in the Gp3 (Tables 1 and 2).

Table 1. Complete blood count of all experimental groups for 12 weeks.

\begin{tabular}{lllll}
\hline \multicolumn{1}{c}{ Groups } & \multicolumn{1}{c}{ Hb $(\mathbf{g} / \mathbf{d l})$} & \multicolumn{1}{c}{$\mathbf{R B C s}(\times \mathbf{1 0} / \boldsymbol{\mu l})$} & \multicolumn{1}{c}{ Hct $(\boldsymbol{\%})$} & \multicolumn{1}{c}{ Plt $(\times \mathbf{1 0} / \mathbf{\mu l})$} \\
\hline -Ve control & $12.5 \pm 1.36^{\mathbf{a}, \mathbf{b}}$ & $5.95 \pm 1.54$ & $39.3 \pm 6.19$ & $623 \pm 168.9$ \\
STZ alone & $12.26 \pm 0.54^{\mathbf{a}, \mathbf{b}}$ & $6.72 \pm 0.73$ & $37.4 \pm 1.52$ & $979.6 \pm 257$ \\
DEN alone & $12.46 \pm 1.13^{\mathbf{a}, \mathbf{b}}$ & $7.20 \pm 0.81$ & $40.86 \pm 1.49$ & $586.8 \pm 23.2$ \\
Met Alone & $11.62 \pm 1.97^{\mathbf{b}}$ & $6.58 \pm 1.78$ & $35.98 \pm 7.29$ & $702.2 \pm 269$ \\
STZ/Met & $13.2 \pm 0.98^{\mathbf{a}, \mathbf{b}}$ & $6.9 \pm 1.26$ & $39.1 \pm 2.97$ & $970.6 \pm 157$ \\
DEN/Met & $15.88 \pm 1.49^{\mathbf{a}}$ & $8.45 \pm 0.95$ & $42.76 \pm 3.25$ & $1135.4 \pm 747$ \\
F Value & 3.89 & 1.37 & 0.91 & 1.22 \\
$\mathbf{p}$ Value & 0.025 & 0.303 & 0.508 & 0.359 \\
\hline
\end{tabular}

Table 2. Differential leucocytes count of all experimental groups for 12 weeks.

\begin{tabular}{lllll}
\hline Group & WBCs $(\times \mathbf{1 0 3} / \mathbf{\mu l})$ & Neut. $(\%)$ & Lymph. $(\%)$ & Mono. (\%) \\
\hline -Ve control & $10.3 \pm 3.7^{\mathbf{b}}$ & $11.6 \pm 2.408$ & $76.4 \pm 6.39$ & $11.8 \pm 6.76$ \\
STZ alone & $13.4 \pm 1.8^{\mathbf{a}, \mathbf{b}}$ & $9 \pm 5.099$ & $80.6 \pm 7.50$ & $11.8 \pm 3.96$ \\
DEN alone & $25.64 \pm 6.8^{\mathbf{a}}$ & $16.4 \pm 7.83$ & $68.2 \pm 11.12$ & $17.2 \pm 4.32$ \\
Met alone & $10.68 \pm 4.67^{\mathbf{b}}$ & $12 \pm 8.63$ & $73 \pm 11.83$ & $15 \pm 6.96$ \\
STZ/Met & $9.48 \pm 3.09^{\mathbf{b}}$ & $9.2 \pm 5.40$ & $77.6 \pm 7.19$ & $13.4 \pm 2.61$ \\
DEN/Met & $9.3 \pm 5.52^{\mathbf{b}}$ & $8 \pm 2.45$ & $74.8 \pm 9.18$ & $13.4 \pm 2.70$ \\
F-Value & 5.72 & 0.83 & 0.65 & 0.54 \\
p-Value & 0.006 & 0.552 & 0.664 & 0.744 \\
\hline
\end{tabular}




\section{Ahmed Mobasher M et al}

Met protects against the impairment of liver functions induced by STZ and DEN

The biochemical analysis of serum samples showed that levels of ALT, AST, TB, ALP, and Arginase were increased post-DM induction by STZ (Gp2) (Table 3 and Fig. 3). A similar pattern of these parameters was found after DEN injection to induce $\mathrm{HCC}$ in rats $(\mathrm{Gp} 3)$. Treatment with Met post-STZ or DEN injections (Gp5 and Gp6) resulted in a decrease in ALT, AST, TB, ALP, and arginase levels. However, treatment with Met alone resulted in a marginal increase in the levels of ALP and arginase (Fig. 3). The levels of TP and albumin decreased post-DM induction by STZ (Gp2) and HCC induction by DEN injection (Gp3). However, Met treatment post-STZ or DEN injections (Gp5 and Gp6) led to reduction in the levels of ALT, AST, TB, ALP and improve the synthetic function of liver by increasing total protein and albumin levels (Table 3 and Fig. 3).

Table 3. Serum alanine transaminase (ALT), aspartate transaminase (AST), total bilirubin (TB), total protein (TP) and albumin (Alb) levels.

\begin{tabular}{|c|c|c|c|c|c|}
\hline Groups & $\operatorname{ALT}(\mathbf{U} / L)$ & $\operatorname{AST}(\mathbf{U} / \mathbf{L})$ & TB (mg/dl) & $\mathbf{T P}(\mathrm{g} / \mathrm{dl})$ & Alb. (g/dl) \\
\hline -Ve control & $58 \pm 5.5^{d}$ & $135 \pm 8.5^{\mathrm{c}}$ & $0.33 \pm 0.03^{d}$ & $5.3 \pm 0.28^{\mathrm{a}}$ & $2.87 \pm 0.24^{\mathrm{a}}$ \\
\hline STZ alone & $88 \pm 3.06^{\mathbf{a}, \mathbf{b}}$ & $207 \pm 11.5^{\mathrm{a}}$ & $0.69 \pm 0.06^{\mathrm{a}}$ & $4.12 \pm 0.24^{b, c}$ & $1.43 \pm 0.13^{b}$ \\
\hline DEN alone & $94 \pm 6.91^{\mathrm{a}}$ & $211 \pm 9.5^{\mathrm{a}}$ & $0.61 \pm 0.06^{\mathbf{a}, \mathbf{b}}$ & $3.68 \pm 0.21^{\mathrm{c}}$ & $1.56 \pm 0.22^{b}$ \\
\hline Met alone & $65 \pm 5.01^{\mathrm{c}, \mathrm{d}}$ & $155 \pm 8.5^{\mathbf{b , c}}$ & $0.33 \pm 0.04^{\mathrm{d}}$ & $4.83 \pm 0.26^{\mathrm{a}}$ & $2.7 \pm 0.12^{\mathrm{a}}$ \\
\hline STZ/Met & $69 \pm 3.6^{\text {c,d }}$ & $170 \pm 7.51^{b}$ & $0.43 \pm 0.06^{\mathbf{c}, \mathbf{d}}$ & $5.01 \pm 0.27^{\mathrm{a}}$ & $2.46 \pm 0.25^{\mathrm{a}}$ \\
\hline $\begin{array}{l}\text { DEN/Met } \\
\text { F-Value }\end{array}$ & $\begin{array}{l}78 \pm 5.29^{\mathbf{b}, \mathbf{c}} \\
22.61\end{array}$ & $\begin{array}{l}167 \pm 8.91^{b} \\
31.54\end{array}$ & $\begin{array}{l}0.53 \pm 0.05^{\mathbf{b}, \mathbf{c}} \\
25.23\end{array}$ & $\begin{array}{l}4.73 \pm 0.23^{\mathrm{a}, \mathrm{b}} \\
17.39\end{array}$ & $\begin{array}{l}1.63 \pm 0.098^{b} \\
35.13\end{array}$ \\
\hline & 0.000 & 0.000 & 0.000 & 0.000 & 0.000 \\
\hline
\end{tabular}

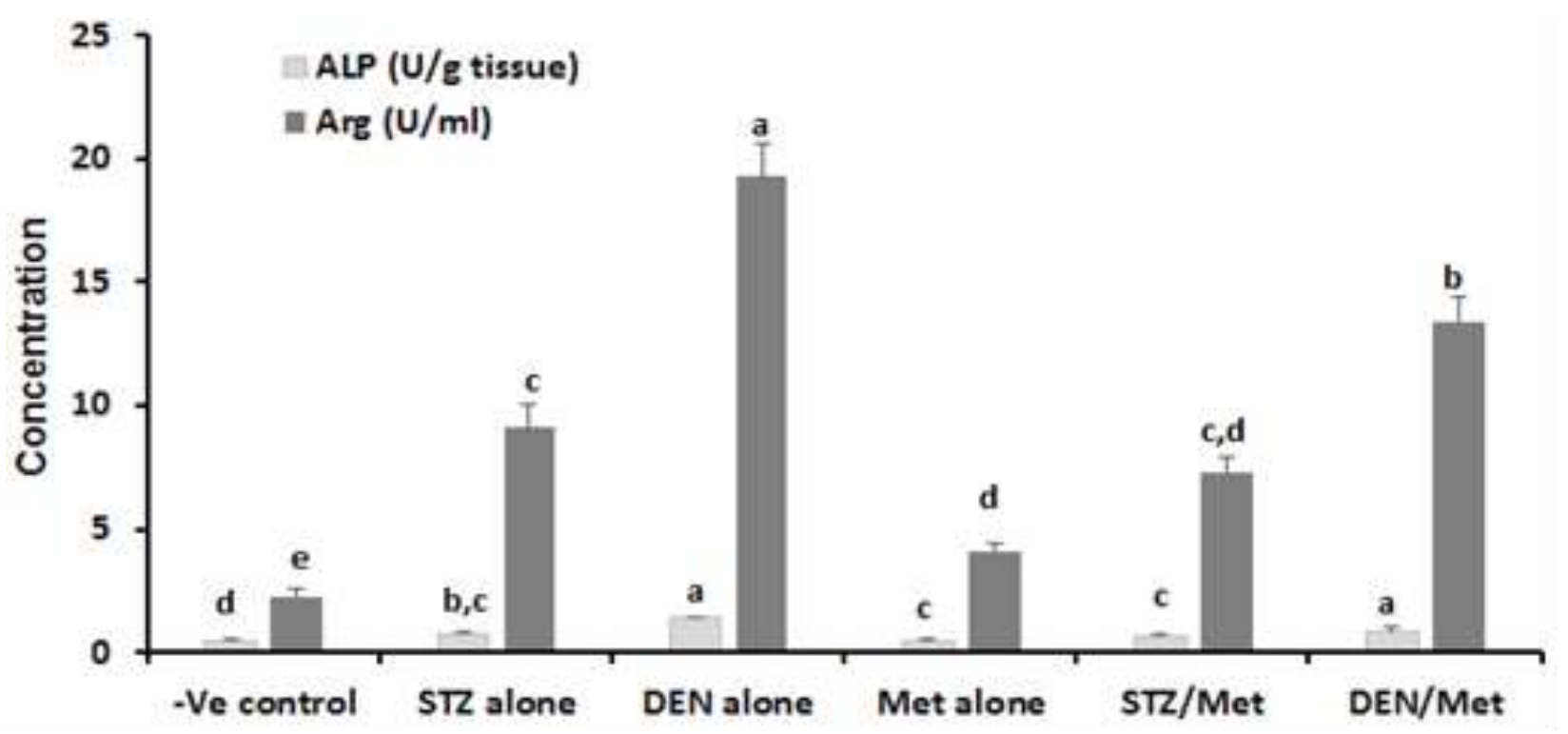

Fig. 3. ALP and arginase levels of control, DM and HCC-induced rats. The results display the mean of seven rats per group under the different treatment conditions. Bars represent standard deviation. Columns with different lower-case letters indicate significant difference between treated groups and the control at $\mathrm{p}<0.05$ (Tukey's test). 
Met attenuates the elevation of lipid profile and enhances the reduction of the antioxidant biomarkers induced by STZ and DEN

Cholesterol, triglycerides, and LDL levels in serum samples from rats, which injected with STZ or DEN were increased while HDL levels were decreased (Gp2 and Gp3). The treatment with Met post-STZ or DEN injections; however, significantly declined the levels of these parameters when compared with STZ or DEN injected groups (Fig. 4). HDL levels were slightly increased only in the STZ/Met group when compared with STZ alone, but not changed in DEN/Met group as compared to DEN alone (Fig. 4).

Furthermore, induction of DM with STZ or induction of HCC with DEN (Gp2 and Gp3) led to an increase in the levels of hepatic MDA concomitant with a decrease in the levels of SOD, CAT, and GSH. Treating diabetic and HCCbearing rats with Met lead to a significant increase in the levels of CAT and GSH while SOD levels were not affected. A significant decrease in MDA levels (Gp5 and Gp6) when compared to their controls (Gp2 and Gp3) (Figs. 5A-5B) was also noticed in these groups.

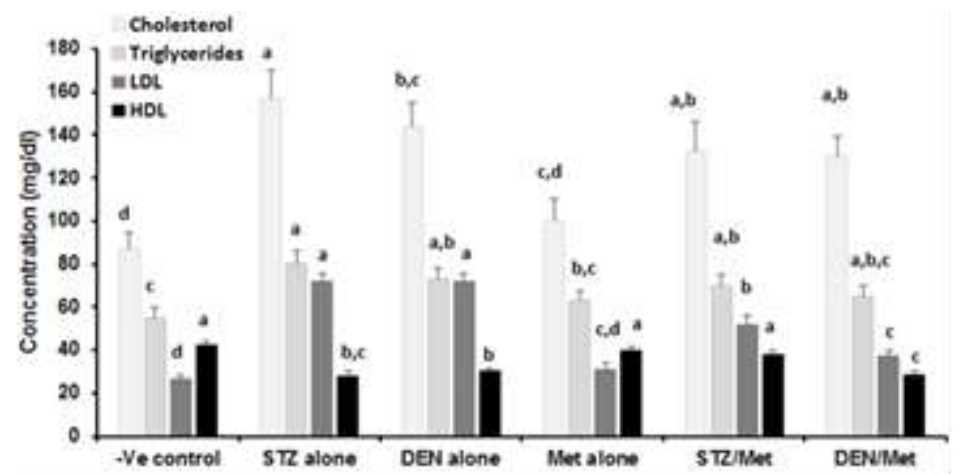

Fig. 4. Lipid profile of control, DM, and HCC-induced rats. The results are mean of seven rats per group under different treatment conditions. Bars represent standard deviation. Columns with different lower-case letters indicate significant difference between treated groups and the control at $\mathrm{p}<0.05$ (Tukey's test).

\section{Met improved liver histology in DM and HCC-rats}

Light microscopic examination of control liver sections revealed normal hepatic architecture with hepatic strands radiating from the central vein. In addition, the polyhedral hepatocytes appeared with homogeneous acidophilic cytoplasm and a large central nucleus and anastomosing strands of hepatocytes were separated with blood sinusoids. Likewise, in the livers from control rats the central vein lined with endothelial cells (Fig. 6A). The STZ-treated group (Gp2) showed marked histological changes manifested by disrupted lobular changes with cellular inflammation at the narrow blood sinusoids. Marked degenerative signs and characterized apoptotic cells were also observed (Fig. 6B). The analysis of DEN-treated group (Gp3) revealed marked features of grade 1 HCC characterized by multiple rounded masses of eosinophilic and multivacuolated cytoplasm of hepatocytes

that also showed large rounded vesicular nuclei with prominent nucleoli together with necrotic cells and severe bleeding in the blood sinusoids and portal spaces (Fig. 6C). The Met-treated group (Gp4) recorded slight bleeding in the blood sinusoids and portal spaces while the hepatocytes appeared intact (Fig. 6D). STZ/Met treated group (Gp5) showed some necrotic areas accompanied by typical hepatic architecture, some vacuolated hepatocytes and narrow blood sinusoids (Fig. $6 \mathrm{E})$. Notably, the liver samples of rats treated with DEN and Met preserved the overall liver architecture, and no evidence of major histological injury was found. In these samples, HCC appeared remarkably reduced, but apparent cellular infiltration at the blood sinusoids and trabeculae of large polyhedral hepatocytes have polymorphic nuclei were still present (Fig. 6F). 


\section{Ahmed Mobasher M et al}

A
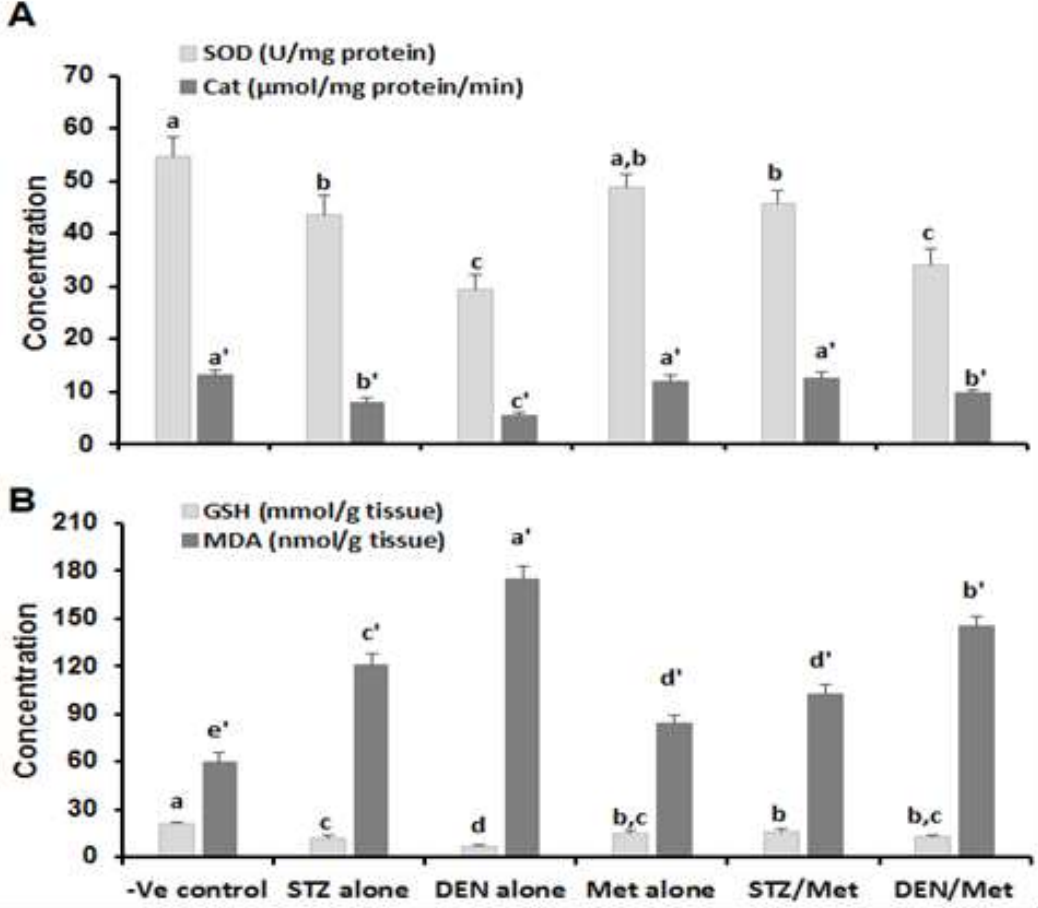

Fig. 5. (A) Superoxide dismutase (SOD) and Catalase, (B) Glutathione (GSH) and Malondialdehyde (MDA) levels as indicators for oxidative stress. The results are mean of seven rats per group under different treatment conditions. Bars represent standard deviation. Columns with different lower-case letters indicate a significant difference between treated groups and the control at $\mathrm{p}<0.05$ (Tukey's test).

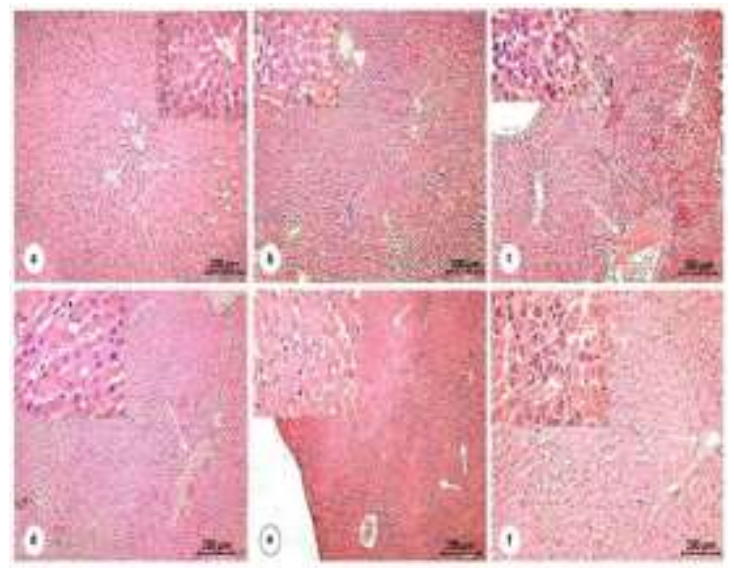

Fig. 6. Hematoxylin \& Eosin stain: photomicrographs of liver sections of rats. (A) control group (Gp1) revealing the hepatic strands (arrows) of the hepatic cells (HC) arising from the central vein (CV), lined by the normal endothelial cells (arrowheads), [X.10, 40]. (B) STZ treated group (Gp2) for 105 days showing some apoptotic cells (thin arrows) and inflammation at the narrowed blood sinusoids and the congestion of the central vein (arrowhead). Hepatocellular degeneration (thick arrows), [X. 10,40]. (C) DEN treated group (Gp3) for 45 days revealing notable features of hepatocellular carcinoma HCC grade 1. The malignant cells consisted of multiple rounded mass of cells with a large amount of eosinophilic multivacuolated cytoplasm (small arrows) and large rounded vesicular nuclei with prominent nucleoli. Additionally, many necrotic sites and severe hemorrhage in the central vein is present [X.4,40]. (D) MET treated group (Gp4) for 105 days showing slight hemorrhage in the blood sinusoids and the portal spaces (arrows). Intact hepatocytes with a normal architecture of the hepatic tissue, [X. 4,40]. (E) STZ/MET treated group (Gp5) in which the rats were treated with STZ for 15 days followed by MET for 90 days, revealed more or less normal hepatic architecture (arrows) and some necrotic areas (thick arrow). The magnified region shows some narrowing blood sinusoids and vacuolation of few hepatocytes (thin arrow), [X. 4, 40]. (F) DEN/MET treated group (Gp6). The rats were treated with DEN for 45 days followed by MET for 60 days revealing apparent cellular infiltration at the vascular area (thin arrow). The appearance of trabeculae of large polyhedral cells (malignant hepatocytes) with polymorphic nuclei and cellular infiltrate blood sinusoids (thick arrow), [X. 10, 40]. 
Met reduced the liver PCNA and caspase-3 immunohistochemical changes in DM and HCC-rats.

Liver sections from the control group displayed standard lobular architecture with negative detectable immunostaining for PCNA (proliferating cell nuclear antibodies) (Fig. 7A). Whereas DEN-treated group (Gp3) showed apparent high immunostaining for PCNA, particularly around the central vein (Fig. 7C), treatment with STZ (Gp2) revealed very few positively stained nuclei in the Hepatic tissue (Fig. 7B). Also, treatment with Met alone showed higher apparent scattered positively stained nuclei compared to the STZ-treated group (Fig. 7D). Group of rats which received DEN followed by Met treatment resulted in decreased PCNA immunostained cells, which were mainly localized in the pericentral area (Fig. 7F).
Group of rats which received STZ followed by Met completely lost the positive immunostaining for PCNA (Fig. 7E).

Control liver sections revealed negative immunostaining for active caspase-3 (Fig. 8A). In sections of STZ treated group (Gp2) elevation of immunolabelled cells for active caspase-3 was markedly observed in many areas of the hepatic tissue (Fig. 8B). Of note, DEN-treated group (Gp3) showed the most abundance of immunostained apoptotic cells that appeared surrounding the congested central vein (Fig. 8C). Met-treated group (Gp4) showed almost no immunostained cells, but revealed some apoptotic cells concentrated at the portal spaces (Fig. 8D). Importantly, STZ/Met and DEN/Met treated groups (Gp5 and Gp6) showed reductions in immunostained active caspase-3 apoptotic cells (Figs. 8E-8F).

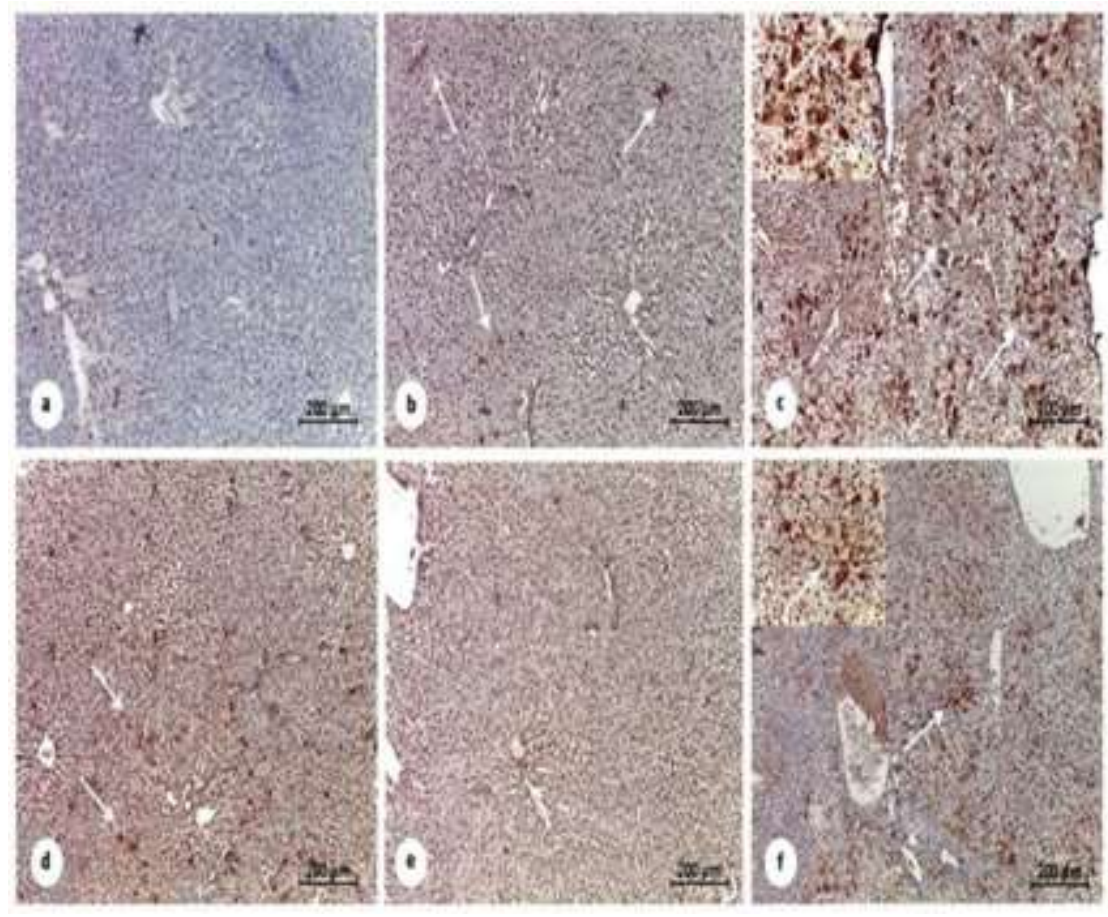

Fig. 7. Photomicrographs of the immunohistochemical stain of proliferating cell nuclear antigen PCNA in the liver of control and treated rats. Counterstained blue nuclei of the hepatocytes are negatively expressed for PCNA in the control untreated group (Gp1) [Fig. (A) X40]. Many proliferating tumor cells show strong nuclear staining of PCNA in DEN treated group (Gp3) at many sites (thin and thick arrows) [Fig. (C) X100]. Few nuclei of hepatocytes (arrows) show PCNA expression in the STZ treated group (Gp2) [Fig. (B)X100] and in the STZ/MET treated group (Gp5) [Fig. (E) X:100. MET treated group (Gp4) showing mild PCNA expression (arrows) [Fig. (D) X100]. DEN/MET treated group (Gp6) shows mild expression of PCNA (arrows) and receding of the tumor cells, which become fewer and decreased intensity [Fig. (F) X100]. 


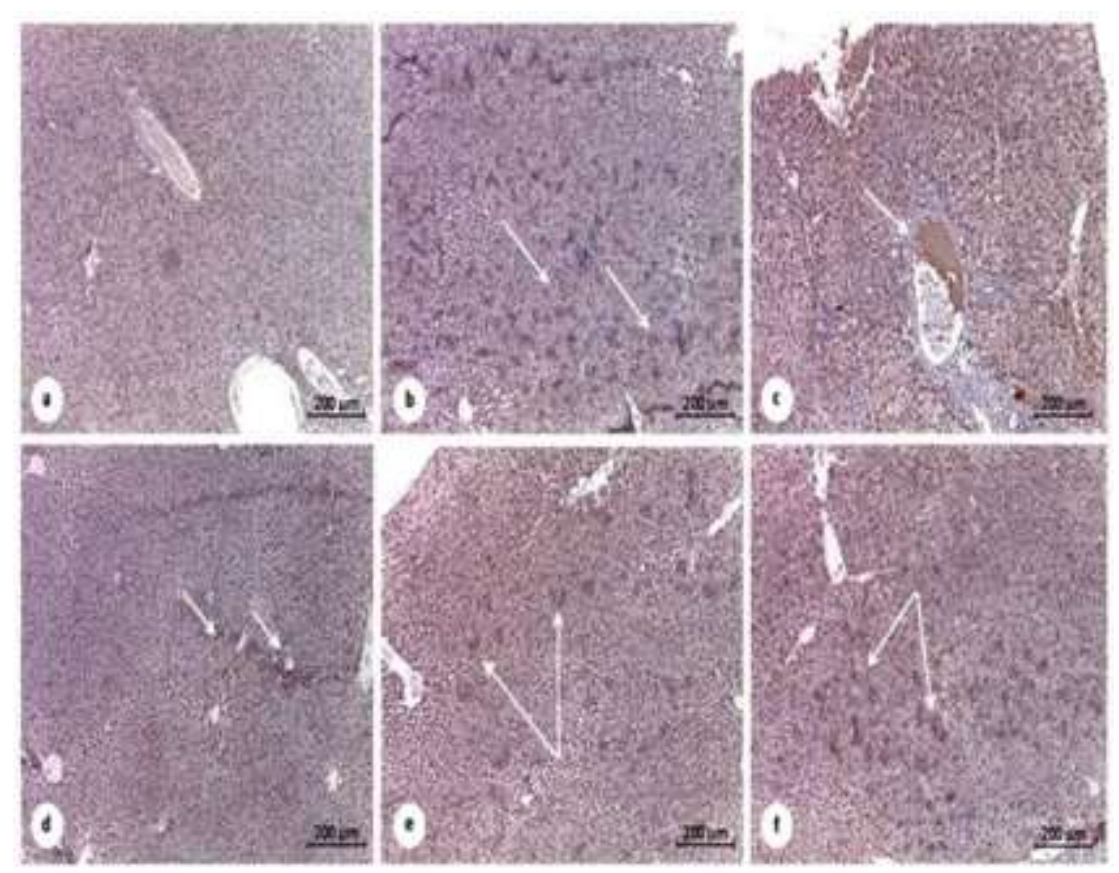

Fig. 8. Photomicrographs of immunohistochemical apoptotic cell population marker Caspase-3 in the liver of control and treated rats. Counterstained nuclei of the hepatocytes are negatively expressed for Caspase in the control untreated group (Gp1) [Fig (A) X40]. Significantly high expression of the apoptotic marker observed in STZ treated group (Gp2) at many sites (arrows) [Fig (B) X40]. The most expressed apoptotic cells are surrounding the congested central vein (arrow) in DEN-treated group (Gp3) [Fig (C)X40]. MET treated group (Gp4) [Fig (D) X:40] show poor expression of Caspase -3 antibodies, but display some apoptotic cells concentrated at the portal space (arrows). ZET/MET treated group (Gp5) shows mild Caspase expression (arrows) [Fig (E) X40]. DEN/MET treated group (Gp6) shows mild expression of Caspase (arrows) as various populations become fewer and less intensity than in STZ treated group (Gp2) [Fig (F) X40].

\section{Discussion}

DM is one of the most common diseases all over the world. Notably, several studies revealed that diabetes might lead to the initiation of HCC upon exposure of the tissues to the generated oxidative stress (35). These previous findings prompted us to design this study to address the impact of Met, the commonly used therapeutics to treat DM, on the oxidative status of diabetic and HCC-bearing rats. Our data showed that injection with STZ or DEN led to a significant no increase in body weights after 14 weeks' post-injection. Moreover, the treatment with Met, post-induction of diabetes or HCC did not increase total body weight. As expected, Met treatment post-STZ injection reduced the levels of glucose but glucose levels were not altered upon Met treatment in either control or DEN-injected groups.

Researchers pointed out that Met not only reduces blood glucose levels and improves diabetics' prognosis, but also decreases oxidative stress and the production of proinflammatory cytokines (36). The elevated glucose levels inside the mitochondria lead to an increase in electron transport chain activity, which in turn, increases the levels of superoxide anion production and oxidative stress (37). Therefore, a higher levels of generated superoxide radical expected to be seen in rats suffered from DM. Met acts as an antioxidant agent to remove these generated free radicals and inhibits ROS production by decreasing NADPH oxidase expression, a family of enzymes that increase the progression of many disease complications.

Maintaining cellular redox homeostasis is a major challenge in any biological system to prevent lipid peroxidation, membrane damage and DNA mutations (38). Upon exposure to the released free radicals, the lipid layer in the cell membrane undergoing peroxidation and oxidative destruction of polyunsaturated fatty acids (arachidonic acid) of the cell membranes, which have dire consequences effects on cellular functions. Malondialdehyde (MDA) is one of the toxic and reactive aldehyde metabolites released 
because of this membrane destruction (38). Nowadays, MDA is the most frequently used biomarker of oxidative stress in many health conditions. Our results are in concordance with such a notion; show significant increases in hepatic MDA concentration in diabetic rats, corroborating the previous work of Erejuwa et al. who reported that lipid peroxidation was significantly elevated while body weight was reduced in diabetic rats (39). On the contrary, treating diabetic and HCC-bearing rats with a combination of MET and STZ or DEN decreased the hepatic MDA levels in these treated groups. These results indicate that Met display antiperoxidative influence that could be because of its hypoglycemic effect as mentioned before (40).

$\mathrm{GSH}$, one of the major endogenous low molecular weight antioxidants that serve as the most abundant cellular thiol resource and provides a buffer system to maintain cellular redox status mainly in diabetic patients. GSH reduces $\mathrm{H}_{2} \mathrm{O}_{2}$ through glutathione peroxidase ( $\mathrm{Gpx}$ ) then being oxidized to its disulfide form (GSSG) (41). Decreased GSH levels were previously published in diabetic rats after treatment with STZ (42), which could suggest that the glutathione defense system was significantly compromised. The elevated MDA levels in diabetic rats might also share in the impairment of glutathione-mediated defense mechanisms as previously reported (42). This was in agreement with our results, which show an increase in the levels of hepatic MDA concomitant with a decrease in the levels of GSH with STZ and DEN treatment. Our data confirmed that treating diabetic and HCC-bearing rats with Met lead to a significant increase in the levels of GSH.

Undoubtedly, SOD is essential in the control of oxidative stress in DM (43) and high SOD activity was noticed as a response of amplified superoxide anions generation, and this higher activity was duo to the increased turnover of $\mathrm{H}_{2} \mathrm{O}_{2}$. Unfortunately, as a response to these elevated levels, CAT, an antioxidant enzyme that usually converts $\mathrm{H}_{2} \mathrm{O}_{2}$ to $\mathrm{H}_{2} \mathrm{O}$ and $\mathrm{O}_{2}$, is incapable to counteract theses elevated levels of $\mathrm{H}_{2} \mathrm{O}_{2}$, and was inhibited. Hence, all these effects might be the cause of diminished CAT activity noticed in diabetics (43). This was in agreement with our results, which show an obvious decrease in the levels of SOD, CAT with STZ and DEN treatment. While, treating diabetic and HCCbearing rats with Met lead to a significant increase in the levels of CAT.

Our investigation showed that diabetes induction by STZ or HCC induction by DEN caused an elevation in the level of liver enzymes such as AST, ALT, ALP, arginase. Similarly, our data revealed many histopathological alterations in hepatic tissues after treatment with STZ or DEN. Additionally, both the immunohistochemical markers (PCNA and caspase-3) expressions in the hepatic tissues exhibited consistent confirmatory results with the biochemical and histopathological findings in all treated groups. Our study demonstrated that treatment with Met after the induction of DM or HCC provided such kind of protection in the liver by decreasing the levels of liver transaminases (ALT and AST) levels in serum. Interestingly, cotreatment of Met with STZ or DEN decreased the evidence of hepatic histological injury and led to a remarkable reduction of HCC features in the liver tissue, although, moderate cell infiltration was recorded. Despite the accumulation of a large body of data, the protective effect of Met is still under study. This effect is assumed to be via inhibition of apoptosis, improvement of mitochondrial dysfunction, decrease in oxidative stress, and suppression of NF-Kb (nuclear factor kappa-light-chain-enhancer of activated B-cells)mediated inflammation. Since Met is positively charged, it enters into the mitochondria of hepatocytes and inhibits ATP production, which in turn, results in the acute inhibition of gluconeogenesis. Also, it was previously reported that Met suppresses liver inflammation by inhibiting interleukin-6-stimulated inflammatory response via activation of AMP-activated protein kinase (AMPK) (44). Moreover, Met may promote the stabilization of phospholipid in the cell membrane thereby preserving cell integrity, and ultimately prevents the cellular escape of cytoplasmic components and enzymes from the hepatocytes into the bloodstream. The membranestabilizing property of Met will subsequently prevent the necrosis of the hepatic cells (45). In addition, an essential metabolic impact of Met in 
cancer cells is the hindrance of mitochondrial complex I prompting an unusual increment in the progression of electrons towards oxygen and producing ROS (45). Fu et al., (46) can interpret the previous finding, who reported that the metformin treatment significantly accelerates the production of ROS and reduced mitochondrial membrane potential, causing cell death via DNA damage-induced apoptosis. Additionally, Met exhibits anticancer activity via ROS production potentially leading to apoptosis. At the molecular level, Met primarily functions via the activation of the AMPK- dependent pathway to trigger cell death even when the suppression of mitochondrial complex I does not increase ROS production (47).

Taken together, the results of the present study

\section{References}

1. Lam DW, LeRoith D. The worldwide diabetes epidemic. Curr Opin Endocrinol Diabetes Obes. 2012;19(2):93-96.

2. Benedict M, Zhang X. Non-alcoholic fatty liver disease: An expanded review. World J Hepatol. 2017;9(16):715-732.

3. Noureddin M, Rinella ME. Nonalcoholic fatty liver disease, diabetes, obesity, and hepatocellular carcinoma. Clin Liver Dis. 2015;19(2):361-79.

4. Abdullaev FI, Rivera LR, Roitenburd BV, Espinosa AJ. Pattern of childhood cancer mortality in Mexico. Arch Med Res. 2000;31(5):526-31.

5. Seeff LB, Hoofnagle JH. Epidemiology of hepatocellular carcinoma in areas of low hepatitis $\mathrm{B}$ and hepatitis $\mathrm{C}$ endemicity. Oncogene. 2006;25(27):3771-7.

6. Huang Y, Cai X, Qiu M, Chen P, Tang H, Hu $\mathrm{Y}$, et al. Prediabetes and the risk of cancer: a metaanalysis. Diabetologia. 2014;57(11):2261-9.

7. Mantovani A, Targher G. Type 2 diabetes mellitus and risk of hepatocellular carcinoma: spotlight on nonalcoholic fatty liver disease. Ann Transl Med. 2017;5(13):270.

8. Tan Y, Wei S, Zhang W, Yang J, Yang J, Yan L. Type 2 diabetes mellitus increases the risk of hepatocellular carcinoma in subjects with chronic hepatitis B virus infection: a meta-analysis and systematic review. Cancer Manag Res. 2019;11:705-713.

9. DeCensi A, Puntoni M, Goodwin P, Cazzaniga M, Gennari A, Bonanni B, et al. Metformin and reinforce the protective role of Met when used as a treatment against DM or HCC. Met holds a great promise in the improvement of the histology of hepatic tissue and improved the liver functions. These data have important clinical implications for designing therapeutic protocols toward DM and HCC diseases that will help to control disease development and therapy, suggesting this drug as a novel therapeutic option against oxidative stress induced by DM or HCC in the hepato-renal axis.

\section{Acknowledgment}

This study was funded by a grant from Deanship for scientific Research, Jouf University, Sakaka, Saudi Arabia (Grant no. 579/39).

The authors declare no conflict of interest.

cancer risk in diabetic patients: a systematic review and metaanalysis. Cancer Prev Res (Phila). 2010;3(11):1451-61.

10. Giovannucci E, Harlan DM, Archer MC, Bergenstal RM, Gapstur SM, Habel LA, et al. Diabetes and cancer: a consensus report. Diabetes Care. 2010;33(7):1674-85.

11. Negre-Salvayre A, Auge N, Ayala V, Basaga H, Boada J, Brenke R, et al. Pathological aspects of lipid peroxidation. Free Radic Res. 2010;44(10):1125-71.

12. Halliwell B. Free radicals and antioxidantsquo vadis? Trends Pharmacol Sci. 2011;32(3):125-30.

13. Nathan DM, Buse JB, Davidson MB, Ferrannini E, Holman RR, Sherwin R, et al. Medical management of hyperglycaemia in type 2 diabetes mellitus: a consensus algorithm for the initiation and adjustment of therapy: a consensus statement from the American Diabetes Association and the European Association for the Study of Diabetes. Diabetes Care. 2009;32(1):193-203.

14. Bosetti C, Franchi M, Nicotra F, Asciutto R, Merlino L, La Vecchia C, et al. Insulin and other antidiabetic drugs and hepatocellular carcinoma risk: a nested case-control study based on Italian healthcare utilization databases. Pharmacoepidemiol Drug Saf. 2015;24(7):771-8. 15. DePeralta DK, Wei L, Ghoshal S, Schmidt B, Lauwers GY, Lanuti M, et al. Metformin prevents hepatocellular carcinoma development 
by suppressing hepatic progenitor cell activation in a rat model of cirrhosis. Cancer. 2016;122(8):1216-27.

16. Gallagher EJ, LeRoith D. Diabetes, cancer, and metformin: connections of metabolism and cell proliferation. Ann N Y Acad Sci. 2011;1243(1):54-68.

17. Alzoubi KH, Khabour OF, Al-Azzam SI, Tashtoush NH, Mhaidat NM. Metformin eased cognitive impairment induced by chronic Lmethionine administration: potential role of oxidative stress. Curr Neuropharmacol. 2014;12(2):186-92.

18. Brondum E, Nilsson H, Aalkjaer C. Functional abnormalities in isolated arteries from GotoKakizaki and streptozotocin-treated diabetic rat models. Horm Metab Res. 2005;37(Suppl 1):56-60.

19. Salemi Z, Rafie E, Goodarzi MT, Ghaffari MA. Effect of Metformin, Acarbose and Their Combination on the Serum Visfatin Level in Nicotinamide/Streptozocin-Induced Type 2 Diabetic Rats. Iran Red Crescent Med J. 2016;18(3):e23814.

20. Kaplan RM. The connection between clinical health promotion and health status: A critical overview. Am Psychol. 1984,39(7):755-65.

21. Belfield A, Goldberg DM. Revised assay for serum phenyl phosphatase activity using 4-aminoantipyrine. Enzyme. 1971;12(5):561-73.

22. Patil NB, Somvanshi BS, Kothari RM. A simple and rapid high recovery protocol for the purification of arginase. Biotechnol Tech. 1990;4(2):133-36.

23. Lowry OH, Roserbrough NJ, Farr AL, Randall RJ. Protein measurement with the Folin phenol reagent. J Biol Chem. 1951;193(1):265-75.

24. Burtis C, Bruns D. Tietz fundamentals of clinical chemistry. 6th ed. Elsevier Health Sciences; 2007.

25. Walter M, Gerade H. A colorimetric method for determination bilirubin in serum and plasma. Micro Chem J. 1970;15:231-36.

26. Allain CC, Poon LS, Chan CS, Richmond W, $\mathrm{Fu}$ PC. Enzymatic determination of total serum cholesterol. Clin Chem. 1974;20(4):470-475.

27. Fossati P, Prencipe L. Serum triglycerides determined colorimetrically with an enzyme that produces hydrogen peroxide. Clin Chem. 1982;28(10):2077-80.

28. Burstein M, Scholnick HR, Morfin R. Rapid method for the isolation of lipoproteins from human serum by precipitation with polyanions. $\mathrm{J}$ Lipid Res. 1970;11(6):583-95.

29. Friedewald WT, Levy RI, Fredrickson DS. Estimation of the concentration of low-density lipoprotein cholesterol in plasma, without use of the preparative ultracentrifuge. Clin Chem. 1972;18(6):499-502.

30. Paoletti F, Mocali A. Determination of superoxide dismutase activity by purely chemical system based on $\mathrm{NAD}(\mathrm{P}) \mathrm{H}$ oxidation. Methods Enzymol. 1990;186:209-20.

31. Aebi, H. Catalase in Vitro. Methods Enzymol. 1984;105:121-6.

32.Paglia DE, Valentine WN. Studies on the quantitative and qualitative characterization of erythrocyte glutathione peroxidase. J Lab Clin Med. 1967;70(1):158-69.

33. Li XY, Chow CK. An improved method for the measurement of malondialdehyde in biological samples. Lipids. 1994;29(1):73-75.

34. Bancroft JD, Stevens A. Theory and practical of histological techniques. 4th ed. Churchill Livingstone. New York: Edinburg and London;1996.

35. Matsuzawa-Nagata N, Takamura T, Ando H, Nakamura S, Kurita S, Misu H, et al. Increased oxidative stress precedes the onset of high-fat dietinduced insulin resistance and obesity. Metabolism. 2008;57(8):1071-7.

36. Bułdak L, Łabuzek K, Bułdak RJ, Kozłowski M, Machnik G, Liber S, et al. Metformin affects macrophages' phenotype and improves the activity of glutathione peroxidase, superoxide dismutase, catalase and decreases malondialdehyde concentration in a partially AMPK-independent manner in LPS-stimulated human monocytes/macrophages. Pharmacol Rep. 2014;66(3):418-29.

37. Wiernsperger NF. Oxidative stress as a therapeutic target in diabetes: revisiting the controversy. Diabetes Metab. 2003;29(6):579-85. 38. Vuppalanchi R, Juluri R, Bell LN, Ghabril M, Kamendulis L, Klaunig JE, et al. Oxidative stress in chronic liver disease: relationship between 
peripheral and hepatic measurements. Am J Med Sci. 2011;342(4):314-317.

39. Erejuwa OO, Gurtu S, Sulaiman SA, Ab Wahab MS, Sirajudeen KN, Salleh MS. Hypoglycemic and antioxidant effects of honey supplementation in streptozotocin-induced diabetic rats. Int J Vitam Nutr Res. 2010;80(1):74-82.

40. Liu Z, Li J, Zeng Z, Liu M, Wang M. The antidiabetic effects of cysteinyl metformin, a newly synthesized agent, in alloxan- and streptozocininduced diabetic rats. Chem Biol Interact. 2008;173(1):68-75.

41. Meister A, Anderson ME. Glutathione. Annu Rev Biochem. 1983;52:711-60.

42. Maritim AC, Sanders RA, Watkins JB. Diabetes, oxidative stress and antioxidants: a review. J Biochem Mol Toxicol. 2003;17(1):24-38. 43. Erejuwa OO, Sulaiman SA, Wahab MS, Salam SK, Salleh MS and Gurtu S. Comparison of antioxidant effects of honey, glibenclamide, metformin, and their combinations in the kidneys of streptozotocin-induced diabetic rats. Int J Mol Sci. 2011;12(1):829-843.

44. Ahmad A, Alkreathy HM. Comparative biochemical and histopathological studies on the efficacy of metformin and Nigella sativa oil against thioacetamide-induced acute hepatorenal damage in rats. Biomed Res. 2018;29(15):3106-16.

45. Hadi NR, Al-Amran FG, Swadi A. Metformin ameliorates methotrexate-induced hepatotoxicity. J Pharmacol Pharmacother. 2012;3(3):248-253.

46. Fu YL, Zhang QH, Wang XW, He H. Antidiabetic drug metformin mitigates ovarian cancer SKOV3 cell growth by triggering $\mathrm{G} 2 / \mathrm{M}$ cell cycle arrest and inhibition of $\mathrm{m}$ TOR/PI3K/Akt signaling pathway. Eur Rev Med Pharmacol Sci. 2017;21(5):1169-1175.

47. Rena G, Hardie DG, Pearson ER. The mechanisms of action of metformin. Diabetol. 2017;60(9):1577-1585. 